\title{
Parâmetros hematológicos de frangos de corte alimentados com ração contendo aflatoxina $B_{1}$ e fumonisina $B_{1}$
}

\author{
Hematological parameters of broiler chicks fed rations \\ containing aflatoxin $B_{1}$ and fumonisin $B_{1}$ \\ Eliana Neire Castiglioni Tessari ${ }^{1}$ Carlos Augusto Fernandes Oliveira ${ }^{2}$ \\ Ana Lúcia Sicchiroli Paschoal Cardoso ${ }^{1}$ David Randolph Ledoux ${ }^{3}$ \\ George Rottinghaus ${ }^{4}$
}

\section{RESUMO}

$O$ objetivo deste trabalho foi avaliar os efeitos da aflatoxina $B_{1}\left(A F B_{1}\right)$ e da fumonisina $B_{1}\left(F B_{1}\right)$ sobre o hemograma e o leucograma de frangos alimentados com ração contendo as toxinas isoladamente e em associação, nos niveis

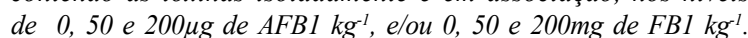
$O$ delineamento experimental foi inteiramente casualizado, em esquema fatorial 3x3, com 9 tratamentos e 12 repetições, totalizando 108 aves. Os frangos foram alimentados com as rações contaminadas do $8^{\circ}$ até o $41^{\circ}$ dia de vida. As aves de todos os grupos alimentados com micotoxinas apresentaram redução $(P<0,05)$ nos valores de hematócrito, hemoglobina $e$ número de hemácias, caracterizando um quadro de anemia, sendo que os grupos mais afetados foram os que receberam as maiores concentrações das toxinas em associação. Observouse um menor $(P<0,05)$ número de leucócitos em todos os tratamentos, porém esta redução foi mais intensa nos grupos tratados com $200 \mu \mathrm{g} \mathrm{kg}^{-1}$ de $A F B_{1}$, com ou sem $F B_{1}$. Conclui-se que a $A F B_{1}$ e a $F B_{l}$, isoladas ou em associação, nos níveis utilizados no estudo, podem afetar negativamente os parâmetros hematológicos de frangos de corte.

Palavras-chave: aflatoxina $B_{l}$, fumonisina $B_{l}$, hematologia aviária, frangos de corte.

\section{ABSTRACT}

The aim of the present study was to evaluate the effects of dietary aflatoxin $B_{1}\left(A F B_{1}\right)$ and fumonisin $B_{I}\left(F B_{1}\right)$ on the hemogram and leucogram of broilers. The mycotoxins were added to rations, singly and in combination, at levels of

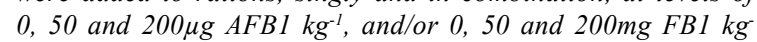

\begin{abstract}
1. A completely randomized $3 \times 3$ factorial design was used, with 9 treatments and 12 replications per treatment (total: 108 birds). Broilers were fed the contaminated rations from dayold 8 to 41. All the mycotoxin-treated groups had decreased $(P<0.05)$ values of hematocrit, hemoglobin concentration and erythrocyte counts, which are common characteristics of anemia. The most affected birds were from groups receiving the highest levels of the combined mycotoxins. Lower $(P<0.05)$ white blood cells counts were also noted in all groups fed mycotoxin-contaminated feeds, however, this reduction was more severe in broilers fed $200 \mu \mathrm{g} \mathrm{AFB} \mathrm{kg}^{-1}$, alone or in association with $F B_{1}$. The conclusion is that both $A F B_{1}$ and $F B_{1}$, singly or in combination at the levels studied, can negatively affect the hematological parameters of broiler chicks
\end{abstract}

Key words: $A F B_{l}, F B_{l}$, avian hematology, broilers.

\section{INTRODUÇÃO}

As micotoxinas são substâncias originadas a partir do metabolismo secundário de fungos de diversos gêneros, os quais se desenvolvem em cereais e derivados amplamente utilizados na alimentação animal (COULOMBE, 1991). As micotoxinas de ocorrência freqüente em nossas condições incluem as aflatoxinas e as fumonisinas.

As aflatoxinas são produzidas por fungos do gênero Aspergillus, espécies A. flavus, $A$. parasiticus e $A$. nomius (MOSS, 1998). São conhecidos,

${ }^{1}$ Pesquisadora Científica, Instituto Biológico, Centro Avançado de Pesquisa Tecnológica do Agronegócio Avícola, Descalvado, SP, Brasil.

${ }^{2}$ Faculdade de Zootecnia e Engenharia de Alimentos, Universidade de São Paulo (USP), Av. Duque de Caxias Norte 225, 13635900, Pirassununga, SP, Brasil. E-mail: carlosaf@usp.br. Autor para correspondência.

${ }^{3}$ College of Agriculture, Food and Natural Resources, University of Missouri, Columbia, MO, United States.

${ }^{4}$ College of Veterinary Medicine, University of Missouri, Columbia, MO, USA. 
atualmente, pelo menos 17 compostos similares designados pelo termo aflatoxina, porém, os principais tipos de interesse médico-sanitário são identificados como $\mathrm{B}_{1}, \mathrm{~B}_{2}, \mathrm{G}_{1}$ e $\mathrm{G}_{2}$, sendo que a aflatoxina $\mathrm{B}_{1}$ (AFB ), além de ser a mais freqüentemente encontrada em cereais, é a que apresenta maior poder toxígeno (COULOMBE, 1991). Um dos principais mecanismos de toxicidade da $\mathrm{AFB}_{1}$ é a inibição da síntese protéica (SANTIN, 2000). Em frangos de corte, a AFB causa depleção linfocítica na medula folicular da bursa de Fabricius (ESPADA et al., 1992) e diminuição das contagens de heterófilos e monócitos (CHANG \& HAMILTON, 1979).

As fumonisinas são produzidas por fungos do gênero Fusarium, principalmente da espécie $\boldsymbol{F}$. moniliforme (verticillioides) (AH-SEO \& WON LEE, 1999). Mais de 16 diferentes tipos de fumonisinas já foram isolados, porém a forma predominante produzida pelo $\boldsymbol{F}$. moniliforme (verticillioides) é a fumonisina $\mathrm{B}_{1}$ $\left(\mathrm{FB}_{1}\right)$, a qual também apresenta maior toxicidade (LEESON et al., 1995). As fumonisinas têm sido associadas com doenças como a leucoencefalomalácia eqüina (LEME) (MEIRELES et al., 1994) e o edema pulmonar suíno (DILKIN et al., 2004). Em aves, níveis de $\mathrm{FB}_{1}$ acima de $150 \mathrm{mg} / \mathrm{kg}$ causam diarréia, diminuição do consumo de alimentos e do ganho de peso, aumento do peso do fígado e rins e necrose hepática (NORRED \&VOSS, 1994)

A ocorrência simultânea de duas ou mais micotoxinas na ração pode determinar a associação dos seus efeitos tóxicos individuais, tal como observado por WEIBKING et al. (1994) e KUBENA et al. (1995) em frangos e perus alimentados simultaneamente com $\mathrm{AFB}_{1}$ e $\mathrm{FB}_{1}$. O objetivo do presente trabalho foi avaliar os parâmetros hematológicos (hemograma, leucograma e contagem diferencial de leucócitos) de frangos alimentados com rações contaminadas com $\mathrm{AFB}_{1} \mathrm{e} / \mathrm{ou}$ $\mathrm{FB}_{1}$.

\section{MATERIAL E MÉTODOS}

Foram utilizados 108 pintos de um dia de idade, com peso médio de $46 \mathrm{~g}$, machos, vacinados no incubatório contra doença de Marek e provenientes de matrizes comerciais de frangos de corte com 53 semanas de idade (linhagem comercial Hybro-PG). As rações experimentais foram formuladas de acordo com os níveis nutricionais recomendados pelo NATIONAL RESEARCH COUNCIL (1994). As aves foram mantidas em um único boxe do $1^{\circ}$ ao $7^{\circ}$ dia de idade, recebendo água e ração ad libitum. No $8^{\circ}$ dia, as aves foram distribuídas aleatoriamente em nove boxes experimentais, com 12 aves em cada. $\mathrm{O}$ delineamento experimental foi inteiramente casualizado, com 9 tratamentos em arranjo fatorial $3 \times 3$, correspondendo a três níveis de incorporação de $\mathrm{AFB}_{1} \mathrm{~kg}^{-1}$ e três níveis de incorporação de $\mathrm{FB}_{1} / \mathrm{kg}$, conforme a tabela 1 . Cada ave foi considerada uma unidade experimental (repetição). Todas as aves receberam as rações experimentais formuladas do 8a ao $41^{\circ}$ dia de vida. Aos 14 dias de idade, as aves foram vacinadas contra a doença de Newcastle, pela via ocular, utilizando-se vacina industrializada (liofilizada) com vírus vivo, tipo $\mathrm{B}_{1}-$ LaSota $^{\mathrm{a}}$.

$\mathrm{A} \mathrm{AFB}_{1}$ e a $\mathrm{FB}_{1}$ utilizadas no experimento foram produzidas no Veterinary Medical Diagnostic Laboratory da Universidade de Missouri, em Columbia, Estados Unidos, a partir do cultivo de cepas toxigênicas de Aspergillus flavus e Fusarium moniliforme (verticillioides), de acordo com OGIDO et al. (2004). A AFB foi produzida em meio de arroz (SHOTWELL et al., 1966) e extraída do meio de cultura utilizando-se clorofórmio. $\mathrm{O}$ extrato foi submetido à evaporação completa do solvente e imediatamente ressuspendido em óleo de milho esterilizado, formando uma suspensão contendo aproximadamente $0,75 \mathrm{mg}$ de $\mathrm{AFB}_{1} \mathrm{~mL}^{-1} \mathrm{e}$ $0,03 \mathrm{mg}$ de aflatoxina $\mathrm{B}_{2} \mathrm{~mL}^{-1}$. $\mathrm{A} \mathrm{FB}_{1}$ foi preparada $\mathrm{e}$ mantida em material de cultivo à base de milho, homogeneizado e esterilizado, conforme os procedimentos descritos por WEIBKING et al. (1993). A concentração de $\mathrm{FB}_{1}$ no material de cultivo foi $6.500 \mathrm{mg} \mathrm{kg}^{-1}$. Neste material, foi também constatada a presença de fumonisina $\mathrm{B}_{2}$, na concentração de $2.100 \mathrm{mg}$ $\mathrm{kg}^{-1}$.

A adição de volumes convenientes da suspensão de AFB (7-30mL $100 \mathrm{~kg}^{-1}$ de ração) e do material de cultivo contendo FB $\left(0,7-3,0 \mathrm{~kg} 100 \mathrm{~kg}^{-1} \mathrm{de}\right.$ ração) foi realizada em um misturador horizontal/ helicoidal (Marconi ${ }^{\circledR}$ ), em cinco diferentes batidas de ração, no decorrer do período experimental, totalizando $550 \mathrm{~kg}$ de ração. A confirmação dos níveis de $\mathrm{AFB}_{1}$ nas rações experimentais foi efetuada através de

Tabela 1 - Níveis de aflatoxina $\mathrm{B}_{1}\left(\mathrm{AFB}_{1}\right)$ e fumonisina $\mathrm{B}_{1}\left(\mathrm{FB}_{1}\right)$ nas rações experimentais, de acordo com $\mathrm{O}$ tratamento.

\begin{tabular}{lcl}
\hline Tratamento & $\mathrm{AFB}_{1}(\mu \mathrm{g} / \mathrm{kg})$ & $\mathrm{FB}_{1}(\mathrm{mg} / \mathrm{kg})$ \\
\hline $\mathrm{T} 1$ & 0 & 0 \\
$\mathrm{~T} 2$ & 0 & 50 \\
$\mathrm{~T} 3$ & 0 & 200 \\
74 & 50 & 0 \\
75 & 50 & 50 \\
$\mathrm{~T} 6$ & 50 & 200 \\
$\mathrm{~T} 7$ & 200 & 0 \\
$\mathrm{~T} 8$ & 200 & 50 \\
$\mathrm{~T} 9$ & 200 & 200 \\
\hline
\end{tabular}


cromatografia de camada delgada, utilizando-se o método descrito por SOARES \& RODRIGUEZ-AMAYA (1989). Para a confirmação dos níveis de FB, utilizouse a técnica de SHEPHARD et al. (1990), com quantificação através de cromatografia líquida de alta eficiência. Após a análise das rações das 5 batidas, os níveis de $\mathrm{AFB}_{1}$ nos tratamentos que continham $50 \mathrm{e}$ $200 \mu \mathrm{g} \mathrm{kg}^{-1}$ foram $46,51 \pm 5,47 \mu \mathrm{g} \mathrm{kg}^{-1}$ e $187,68 \pm 50,28 \mu \mathrm{g}$ $\mathrm{kg}^{-1}$, respectivamente. Para a FB ${ }_{1}$, os níveis encontrados nos tratamentos contendo 50 e $200 \mathrm{mg} \mathrm{kg}^{-1}$ foram $57,27 \pm 1,81 \mathrm{mg} \mathrm{kg}^{-1}$ e $201,00 \pm 25,40 \mathrm{mg} \mathrm{kg} \mathrm{kg}^{-1}$, respectivamente. As rações de cada batida também foram analisadas para a detecção da possível presença de ocratoxina A e zearalenona (SOARES \& RODRIGUEZ-AMAYA, 1989), sendo que nenhuma delas foi detectada nas amostras. Os limites de quantificação do método de análise para ocratoxina $\mathrm{A}$ e zearalenona foram $5,0 \mu \mathrm{g} \mathrm{kg}^{-1}$ e $55,0 \mu \mathrm{g} \mathrm{kg}^{-1}$, respectivamente.

Aos 41 dias de idade das aves, amostras do sangue de 4 aves por tratamento foram colocadas em tubos contendo anticoagulante (ácido etilenodiaminotetraacético), na proporção de $0,1 \mathrm{~mL}$ para $1,0 \mathrm{~mL}$ de sangue, para a realização das análises hematológicas. Os exames da fração celular sanguínea consistiram de: determinação do hematócrito e da hemoglobina, contagem de hemácias, contagem total e diferencial de leucócitos e de trombócitos, interpretação do leucograma e observação da morfologia celular. A determinação do hematócrito foi realizada através do método do microhematócrito, utilizando-se tubo capilar centrifugado a $1.200 \mathrm{~g}$ por 5 minutos, sendo o resultado expresso em porcentagem. A concentração de hemoglobina $\left(\mathrm{g} \mathrm{dL}^{-1}\right)$ foi medida através da técnica de cianometahemoglobina, utilizando-se reagentes do fabricante BIOCLIN, conforme instruções de uso. Para a contagem de hemáceas e leucócitos, utilizou-se uma amostra de sangue e solução de Natt e Herrick's ${ }^{b}$, em uma diluição de 1:200, realizando-se a contagem (N $\mu \mathrm{L}^{-1}$ ) em câmara de Neubauer. A partir dos dados obtidos no hemograma, foram calculados os seguintes índices hematimétricos de Wintrobe: hemoglobina corpuscular média (HCM), expressa em picogramas (pg); concentração de hemoglobina corpuscular média (CHCM), expressa em g dL ${ }^{-1}$; volume corpuscular médio (VCM), expresso em fentolitros (fL).

Para a contagem diferencial leucocitária, preparou-se um esfregaço sanguíneo em lâminas de vidro corado com hematoxilina-eosina. As lâminas foram lavadas com água destilada, secadas ao ar livre e os esfregaços foram observados ao microscópio ótico com objetiva de imersão. A contagem leucocitária foi classificatória para linfócitos, heterófilos, eosinófilos, monócitos e basófilos, calculando-se a proporção de cada tipo em cem células contadas. Adicionalmente, realizou-se a contagem de trombócitos.

Os resultados das análises foram submetidos à análise fatorial $(3 \times 3)$, de acordo com os procedimentos estabelecidos no General Linear Model do SAS $^{\circledR}$ (SAS INSTITUTE, 1992). As médias dos tratamentos que foram estatisticamente diferentes na análise de variância foram analisadas pelo teste de LSD (Least Significant Difference) de Fisher, adotando-se, como nível de rejeição, $\alpha=0,05$.

\section{RESULTADOS E DISCUSSÃO}

Não foram observadas alterações no estado geral das aves durante o período de intoxicação. Os efeitos dos diferentes níveis de $\mathrm{AFB}_{1}$ e $\mathrm{FB}_{1}$, isoladas e associadas na ração, sobre os parâmetros do hemograma dos frangos aos 41 dias de idade, encontram-se na tabela 2. As aves do grupo controle apresentaram todos os valores dentro das faixas de normalidade, de acordo com os dados reportados por NORIEGA(2000) e CARDOSO \& TESSARI (2003). As aves de todos os grupos alimentados com micotoxinas apresentaram redução $(\mathrm{P}<0,05)$ nos valores de hematócrito, hemoglobina e número de hemácias, com exceção do grupo que recebeu somente $50 \mathrm{mg} / \mathrm{kg}$ de $\mathrm{FB}_{1}$, que apresentou número de hemácias semelhante ao controle. Os índices hematimétricos HCM e VCM apresentaram um aumento significativo $(\mathrm{P}<0,05)$ nas aves dos tratamentos de associação $200 \mu \mathrm{g} \mathrm{kg}^{-1}$ de AFB +50mg kg ${ }^{-1}$ de FB $\left(\right.$ T8) e $200 \mu \mathrm{g} \mathrm{kg}^{-1} \mathrm{de}^{\mathrm{AFB}}{ }_{1}+200 \mathrm{mg}$ $\mathrm{kg}^{-1}$ de $\mathrm{FB}_{1}$ (T9), sendo que o VCM também foi maior nos tratamentos $50 \mu \mathrm{g} \mathrm{kg}^{-1} \mathrm{de} \mathrm{AFB}_{1}+200 \mathrm{mg} \mathrm{kg}^{-1} \mathrm{de} \mathrm{FB}^{-}$ (T6) e $200 \mu \mathrm{g} \mathrm{kg}^{-1}$ de $\mathrm{AFB}_{1}$ (T7), quando comparado com os valores obtidos das aves do grupo controle. $\mathrm{O}$ CHCM apresentou-se reduzido $(\mathrm{P}<0,05)$ em todos os tratamentos, com exceção dos grupos alimentados com $50 \mathrm{mg} \mathrm{kg}^{-1}$ de FB ${ }_{1}$ (T2) e $50 \mu \mathrm{g} / \mathrm{kg} \mathrm{de} \mathrm{AFB}_{1}+50 \mathrm{mg} \mathrm{kg}^{-1}$ de $\mathrm{FB}_{1}$ (T5). Estes resultados indicam um quadro de anemia decorrente da intoxicação por $\mathrm{AFB}_{1}$ e $\mathrm{FB}_{1}$, conforme reportado por outros autores (TUNG et al., 1975; JAVED et al., 1995).

O número de hemácias foi acentuadamente reduzido nos grupos que receberam os maiores níveis de micotoxinas, principalmente dos tratamentos de associação $200 \mu \mathrm{g} \mathrm{kg}^{-1} \mathrm{de}_{\mathrm{AFB}}+50 \mathrm{mg} \mathrm{kg}^{-1} \mathrm{de} \mathrm{FB}$ (T8) e $200 \mu \mathrm{g} \mathrm{kg}^{-1} \mathrm{de} \mathrm{AFB}_{1}+200 \mathrm{mg} \mathrm{kg}^{-1} \mathrm{de} \mathrm{FB}_{1}$ (T9), os quais apresentaram menor número de hemácias $(\mathrm{P}<0,05)$ quando comparados aos demais tratamentos. Coerentemente, os valores do hematócrito e de concentração de hemoglobina também diminuíram, 
Tabela 2 - Efeitos da aflatoxina $\mathrm{B}_{1}\left(\mathrm{AFB}_{1}\right)$ e da fumonisina $\mathrm{B}_{1}\left(\mathrm{FB}_{1}\right)$ sobre o hemograma de frangos de corte intoxicados entre 8 e 41 dias de idade $^{1}$.

\begin{tabular}{lccccccc}
\hline $\mathrm{AFB}_{1}$ & $\mathrm{FB}_{1}$ & Hematócrito & Hemoglobina & Hemáceas & HCM & VCM & CHCM \\
$(\mu \mathrm{g} / \mathrm{kg})$ & $(\mathrm{mg} / \mathrm{kg})$ & $(\%)$ & $(\mathrm{g} / \mathrm{dL})$ & $\left(\mathrm{x} 10^{6} / \mu \mathrm{L}\right)$ & $(\mathrm{pg})$ & $(\mathrm{fl})$ \\
\hline 0 & 0 & $43,5 \pm 1,9^{\mathrm{a}}$ & $6,8 \pm 0,07^{\mathrm{a}}$ & $2,5 \pm 0,03^{\mathrm{a}}$ & $27,4 \pm 0,3^{\mathrm{b}}$ & $174,7 \pm 5,8^{\mathrm{c}}$ & $15,7 \pm 0,5^{\mathrm{a}}$ \\
0 & 50 & $36,3 \pm 2,9^{\mathrm{b}}$ & $4,9 \pm 0,35^{\mathrm{b}}$ & $2,3 \pm 0,08^{\mathrm{a}}$ & $21,0 \pm 0,9^{\mathrm{c}}$ & $154,8_{ \pm}+7,3^{\mathrm{c}}$ & $13,5 \pm 0,2^{\mathrm{ab}}$ \\
0 & 200 & $36,7 \pm 0,9^{\mathrm{b}}$ & $4,7 \pm 0,07^{\mathrm{b}}$ & $1,9 \pm 0,03^{\mathrm{b}}$ & $24,1 \pm 0,7^{\mathrm{bc}}$ & $188,9 \pm 2,2^{\mathrm{bc}}$ & $12,8 \pm 0,5^{\mathrm{b}}$ \\
50 & 0 & $36,5 \pm 0,6^{\mathrm{b}}$ & $4,5 \pm 0,29^{\mathrm{b}}$ & $1,9 \pm 0,02^{\mathrm{b}}$ & $23,3 \pm 1,3^{\mathrm{c}}$ & $189,9 \pm 1,8^{\mathrm{bc}}$ & $12,3 \pm 0,7^{\mathrm{b}}$ \\
50 & 50 & $33,7 \pm 2,9^{\mathrm{b}}$ & $4,7 \pm 0,40^{\mathrm{b}}$ & $1,9 \pm 0,01^{\mathrm{b}}$ & $24,5 \pm 2,2^{\mathrm{bc}}$ & $175,8 \pm 5,9^{\mathrm{c}}$ & $13,9 \pm 0,4^{\mathrm{ab}}$ \\
50 & 200 & $33,7 \pm 0,9^{\mathrm{b}}$ & $4,5 \pm 0,27^{\mathrm{b}}$ & $1,6 \pm 0,07^{\mathrm{c}}$ & $28,3 \pm 0,9^{\mathrm{b}}$ & $214,8_{ \pm}+5,7^{\mathrm{b}}$ & $13,2 \pm 0,4^{\mathrm{b}}$ \\
200 & 0 & $29,5 \pm 3,4^{\mathrm{c}}$ & $3,8 \pm 0,29^{\mathrm{c}}$ & $1,3 \pm 0,05^{\mathrm{c}}$ & $28,8 \pm 1,9^{\mathrm{b}}$ & $222,2 \pm 8,3^{\mathrm{b}}$ & $13,0 \pm 1,0^{\mathrm{b}}$ \\
200 & 50 & $35,0 \pm 0,8^{\mathrm{b}}$ & $4,3 \pm 0,18^{\mathrm{b}}$ & $1,2 \pm 0,03^{\mathrm{d}}$ & $37,3 \pm 2,3^{\mathrm{a}}$ & $300,6_{ \pm}+12,5^{\mathrm{a}}$ & $12,4 \pm 0,5^{\mathrm{b}}$ \\
200 & 200 & $26,7 \pm 0,9^{\mathrm{c}}$ & $3,5 \pm 0,20^{\mathrm{c}}$ & $1,1 \pm 0,01^{\mathrm{d}}$ & $32,5 \pm 1,6^{\mathrm{a}}$ & $246,0 \pm 7,6^{\mathrm{ab}}$ & $13,2 \pm 0,3^{\mathrm{b}}$ \\
\hline
\end{tabular}

${ }^{1}$ Resultados expressos em média \pm desvio padrão, para 4 repetições.

$\mathrm{HCM}=$ hemoglobina corpuscular média; $\mathrm{VCM}$ = volume corpuscular médio.

$\mathrm{CHCM}=$ concentração de hemoglobina corpuscular média.

${ }^{\mathrm{a}-\mathrm{c}}$ Médias seguidas de letras distintas, em uma mesma coluna, diferem pelo teste de Fisher $(\mathrm{P}<0,05)$.

sendo que os grupos que receberam $200 \mu \mathrm{g} \mathrm{kg}^{-1}$ de $\mathrm{AFB}_{1}$ (T7) e $200 \mu \mathrm{g} \mathrm{kg}^{-1}$ de $\mathrm{AFB}_{1}+200 \mathrm{mg} \mathrm{kg}^{-1} \mathrm{de} \mathrm{FB}_{1}$ (T9) apresentaram o menor valor $(\mathrm{P}<0,05)$. TUNG et al. (1975) alimentaram frangos com 5,0 e 10,0 $\mu \mathrm{g} \mathrm{g}^{-1} \mathrm{de}$ aflatoxina e observaram que os efeitos sobre os parâmetros hematológicos foram mais pronunciados quanto maior o nível de aflatoxina, similarmente aos resultados do presente estudo.

Com relação aos efeitos isolados da $\mathrm{FB}$ sobre o hemograma de frangos, JAVED et al. (1995) também observaram diminuição do número de hemácias e da concentração de hemoglobina em pintinhos alimentados com FB, em níveis de 125 e $274 \mathrm{mg} \mathrm{kg}^{-1} \mathrm{de}$ ração, no período de 1 a 14 dias de idade. Entretanto, WEIBKING et al. (1993) obtiveram resultados conflitantes, uma vez que não constataram diferenças significativas na contagem de hemácias, concentração de hemoglobina, hematócrito e VCM de frangos de corte alimentados com ração contendo 450 e $525 \mathrm{mg}$ $\mathrm{kg}^{-1}$ de $\mathrm{FB}_{1}$. Resultados discordantes também foram relatados por KUBENA et al. (1997), os quais avaliaram os efeitos da administração de $300 \mathrm{mg}$ de $\mathrm{FB}_{1} \mathrm{~kg}^{-1}$ de ração em perus fêmeas, observando um aumento do número de hemácias. O mecanismo de toxicidade específico pelo qual a $\mathrm{FB}_{1}$ afeta os parâmetros hematológicos não está completamente esclarecido, porém, as discrepâncias encontradas nos resultados de diferentes trabalhos sobre os efeitos das fumonisinas em aves podem estar relacionadas à presença de outros metabólitos de Fusarium no material usado nos experimentos (LEESON et al., 1995).

A tabela 3 apresenta os efeitos dos diferentes níveis de $\mathrm{AFB}_{1}$ e $\mathrm{FB}_{1}$, isoladas e associadas na ração, sobre os parâmetros do leucograma dos frangos aos 41 dias de idade. O número de leucócitos, trombócitos, linfócitos, heterófilos e monócitos obtidos nas aves do grupo controle foram considerados normais, de acordo com NORIEGA (2000) e CARDOSO \& TESSARI (2003). Observou-se uma redução $(\mathrm{P}<0,05)$ no número de leucócitos em todos os tratamentos, quando comparado ao grupo controle, sendo que a redução foi mais intensa nos grupos tratados com $200 \mu \mathrm{g}$ $\mathrm{kg}^{-1}$ de $\mathrm{AFB}_{1}$, com ou sem associação com $\mathrm{FB}_{1}$ (T7, T8 e T9). O mesmo foi observado com relação ao número de linfócitos, heterófilos e monócitos, sendo que os tratamentos de associação $200 \mu \mathrm{g} \mathrm{kg}^{-1} \mathrm{de}_{\mathrm{AFB}}+50 \mathrm{mg}$ $\mathrm{kg}^{-1}$ de FB ${ }_{1}$ (T8) e $200 \mu \mathrm{g} \mathrm{kg}^{-1} \mathrm{de} \mathrm{AFB}_{1}+200 \mathrm{mg} \mathrm{kg}^{-1} \mathrm{de}$ $\mathrm{FB}_{1}$ (T9) apresentaram número de linfócitos e heterófilos ainda menor. Estes resultados indicam um quadro de comprometimento da resposta imunológica mediada por células, típico da exposição de frangos às aflatoxinas em baixas concentrações na ração (ESPADA et al., 1992). O número de trombócitos não diferiu $(\mathrm{P}>0,05)$ entre os tratamentos. Com relação ao número relativo dos tipos celulares, houve um aumento $(\mathrm{P}<0,05)$ no percentual de linfócitos das aves que receberam as micotoxinas, isoladamente ou em associação, com exceção do grupo alimentado com $50 \mathrm{mg} \mathrm{kg}^{-1}$ de $\mathrm{FB}_{1}$ (T2). Este aumento no percentual de linfócitos pode estar relacionado à diminuição da atividade de células fagocitárias causada pela AFB $_{1}$ (CHANG \& HAMILTON, 1979), uma vez que todos os grupos alimentados com ração contendo as micotoxinas apresentaram uma redução $(\mathrm{P}<0,05)$ no percentual de heterófilos, apesar de não ter havido diferença no percentual de monócitos $(\mathrm{P}>0,05)$.

A diminuição do número de leucócitos observada no presente estudo é coerente com os dados 
Tabela 3 - Efeitos da aflatoxina $\mathrm{B}_{1}\left(\mathrm{AFB}_{1}\right)$ e da fumonisina $\mathrm{B}_{1}\left(\mathrm{FB}_{1}\right)$ sobre o leucograma de frangos de corte intoxicados entre 8 e 41 dias de idade $^{1}$

\begin{tabular}{|c|c|c|c|c|c|c|c|c|c|}
\hline \multirow{2}{*}{$\begin{array}{l}\mathrm{AFB}_{1} \\
(\mu \mathrm{g} / \mathrm{kg})\end{array}$} & \multirow{2}{*}{$\begin{array}{c}\mathrm{FB}_{1} \\
(\mathrm{mg} / \mathrm{kg})\end{array}$} & \multirow{2}{*}{$\begin{array}{l}\text { Leucócitos } \\
\left(\times 10^{3} / \mu \mathrm{L}\right)\end{array}$} & \multirow{2}{*}{$\begin{array}{l}\text { Trombócitos } \\
\left(\times 10^{3} / \mu \mathrm{L}\right)\end{array}$} & \multicolumn{2}{|c|}{ Linfócitos } & \multicolumn{2}{|c|}{ Heterófilos } & \multicolumn{2}{|c|}{ Monócitos } \\
\hline & & & & $\left(\mathrm{x} 10^{3} / \mu \mathrm{L}\right)$ & $(\%)$ & $\left(\mathrm{x} 10^{3} / \mu \mathrm{L}\right)$ & $(\%)$ & $\left(\times 10^{3} / \mu \mathrm{L}\right)$ & $(\%)$ \\
\hline 0 & 0 & $24,3 \pm 0,4^{\text {a }}$ & $32,7 \pm 1,0^{\text {a }}$ & $13,5 \pm 0,3^{\text {a }}$ & $49,5 \pm 1,3^{b}$ & $9,7 \pm 0,3^{\text {a }}$ & $47,5 \pm 0,6^{\mathrm{a}}$ & $1,7 \pm 0,2^{\mathrm{a}}$ & $2,7 \pm 1,5^{\text {a }}$ \\
\hline 0 & 50 & $21,7 \pm 0,6^{b}$ & $34,3 \pm 1,5^{\mathrm{a}}$ & $11,1 \pm 0,8^{a}$ & $54,7 \pm 2,5^{b}$ & $11,7 \pm 0,4^{\mathrm{a}}$ & $41,3 \pm 1,0^{\mathrm{b}}$ & $1,2 \pm 0,1^{b}$ & $3,5 \pm 1,3^{\mathrm{a}}$ \\
\hline 0 & 200 & $21,0 \pm 0,6^{b}$ & $31,3 \pm 3,0^{\mathrm{a}}$ & $9,9 \pm 0,6^{\mathrm{b}}$ & $61,0 \pm 3,6^{\mathrm{a}}$ & $9,2 \pm 0,6^{\mathrm{a}}$ & $32,5 \pm 7,2^{\mathrm{c}}$ & $0,7 \pm 0,1^{\mathrm{c}}$ & $6,0 \pm 4,9^{a}$ \\
\hline 50 & 0 & $20,9 \pm 1,3^{b}$ & $32,5 \pm 2,7^{\text {a }}$ & $11,1 \pm 1,1^{\text {a }}$ & $61,5 \pm 2,1^{\mathrm{a}}$ & $6,4 \pm 0,7^{\mathrm{b}}$ & $32,7 \pm 3,6^{c}$ & $0,5 \pm 0,1^{\mathrm{c}}$ & $5,7 \pm 1,7^{\mathrm{a}}$ \\
\hline 50 & 50 & $21,0 \pm 0,9^{\mathrm{b}}$ & $33,3 \pm 3,4^{\mathrm{a}}$ & $9,0 \pm 0,3^{\mathrm{b}}$ & $60,0 \pm 3,2^{\mathrm{a}}$ & $9,0 \pm 0,9^{\mathrm{a}}$ & $33,7 \pm 3,1^{\mathrm{c}}$ & $0,7 \pm 0,2^{\mathrm{c}}$ & $5,2 \pm 1,9^{\mathrm{a}}$ \\
\hline 50 & 200 & $20,2 \pm 0,8^{b}$ & $33,3 \pm 1,7^{\mathrm{a}}$ & $9,8 \pm 0,34^{\mathrm{b}}$ & $59,0 \pm 1,4^{\text {a }}$ & $5,5 \pm 0,4^{\mathrm{b}}$ & $35,0 \pm 1,8^{\mathrm{c}}$ & $0,5 \pm 0,1^{\mathrm{c}}$ & $5,0 \pm 2,0^{\text {a }}$ \\
\hline 200 & 0 & $18,4 \pm 0,5^{c}$ & $37,5 \pm 4,4^{\mathrm{a}}$ & $9,3 \pm 0,5^{\mathrm{b}}$ & $60,0 \pm 3,4^{\mathrm{a}}$ & $5,7 \pm 0,3^{\mathrm{b}}$ & $32,5 \pm 1,3^{\mathrm{c}}$ & $0,4+0,1^{\mathrm{c}}$ & $6,5 \pm 2,5^{\mathrm{a}}$ \\
\hline 200 & 50 & $18,9 \pm 0,5^{\mathrm{c}}$ & $28,7 \pm 3,9^{\mathrm{a}}$ & $7,4 \pm 1,0^{c}$ & $62,3 \pm 4,2^{\mathrm{a}}$ & $4,2 \pm 0,6^{\mathrm{bc}}$ & $35,0 \pm 2,2^{c}$ & $0,4 \pm 0,1^{\mathrm{c}}$ & $4,3 \pm 1,3^{\mathrm{a}}$ \\
\hline 200 & 200 & $17,3 \pm 0,7^{\mathrm{c}}$ & $38,3 \pm 5,7^{\mathrm{a}}$ & $7,4 \pm 0,5^{\mathrm{c}}$ & $63,5 \pm 2,7^{\mathrm{a}}$ & $3,7 \pm 0,6^{\mathrm{c}}$ & $28,0 \pm 2,8^{c}$ & $0,5 \pm 0,1^{\mathrm{c}}$ & $6,7 \pm 1,9^{\mathrm{a}}$ \\
\hline
\end{tabular}

${ }^{1}$ Resultados expressos em média \pm desvio padrão, para 4 repetições.

${ }^{\mathrm{a}-\mathrm{c}}$ Médias seguidas de letras distintas, em uma mesma coluna, diferem pelo teste de Fisher $(\mathrm{P}<0,05)$.

descritos por JAVED et al. (1995), porém contrária aos resultados obtidos por TUNG et al. (1975). Estes autores observaram um aumento no número de leucócitos circulantes de frangos de corte tratados com 5 e $10 \mu \mathrm{g}$ $\mathrm{g}^{-1}$ de $\mathrm{AFB}_{1}$ adicionada à ração, o que parece incoerente à luz dos conhecimentos quanto aos efeitos das aflatoxinas sobre os órgãos hematopoiéticos das aves, sobretudo sobre a bursa de Fabricius (ESPADA et al., 1992), principalmente ao se levar em consideração que os autores utilizaram concentrações 25-50 vezes acima do maior nível utilizado no presente estudo $(200 \mu \mathrm{g}$ $\mathrm{kg}^{-1}$ de $\mathrm{AFB}_{1}$ ). Com relação ao efeito isolado das fumonisinas, BERMUDEZ et al. (1996) também observaram resultados diferentes em perus alimentados com ração contendo $75 \mathrm{mg}$ de $\mathrm{FB}_{1} \mathrm{~kg}^{-1}$, demonstrando um aumento no número total de leucócitos e no número absoluto de linfócitos e heterófilos. Os autores concluíram que os perus são relativamente resistentes à $\mathrm{FB}_{1}$, quando comparados com frangos de corte.

\section{CONCLUSÕES}

Os resultados demonstraram que $\mathrm{AFB}_{1}$ e a $\mathrm{FB}_{1}$, isoladas ou associadas, acarretam um quadro caracterizado por anemia hemolítica, leucopenia, linfopenia e heterofilia em frangos de corte. Desse modo, observa-se que o limite máximo de aflatoxinas em rações $\left(50 \mu \mathrm{g} \mathrm{kg}^{-1}\right)$ adotado pela legislação brasileira (BRASIL, 1988) não pode ser considerado seguro na criação de frangos de corte, uma vez que este nível acarretou alterações em alguns parâmetros hematológicos, sendo, portanto, potencialmente capaz de afetar o desempenho das aves. Os efeitos tóxicos foram mais intensos com a associação entre a $\mathrm{AFB}_{1}$ e a $\mathrm{FB}_{1}$, o que demonstra a necessidade da adoção de limites de tolerância para fumonisinas em rações para frangos de corte.

\section{FONTES DE AQUISIÇÃO}

a NEW VAC-LS, Ford Dodge Saúde Animal Ltda - R. Luiz Fernando Rodriguez, 1701, Campinas, SP.

b IMBRALAB Química e Farmacêutica Ltda - R. Ataulfo Alves, 396, Ribeirão Preto, SP.

\section{REFERÊNCIAS}

AH-SEO, J.; WON LEE, Y. Natural occurrence of the C series of fumonisins in moldy corn. Applied and Environmental Microbiology, v.65, p.1331-1334, 1999.

BERMUDEZ, A.J. et al. The chronic effects of Fusarium moniliforme culture material, containing known levels of fumonisin $\mathrm{B}_{1}$ in turkeys. Avian Diseases, v.40, p.231-235, 1996.

BRASIL. Ministério da Agricultura. Portaria MA/SNAD/SFA n.7, de 9 de novembro de 1988. Diário Oficial da República Federativa do Brasil, Sec.I, p.21.968, 1988.

CARDOSO, A.L.S.P.; TESSARI, E.N.C. Estudo dos parâmetros hematológicos em frangos de corte. Arquivos do Instituto Biológico, v.70, p.419-424, 2003.

CHANG, C.F.; HAMILTON, P.B. Impairment of phagocytosis in chicken monocytes during aflatoxicosis. Poultry Science, v.58, p.562-566, 1979 .

COUlOMBE, R.A. Aflatoxins. In: SHARMA, R.P.; SALUNKHE, D.K. Mycotoxins and phytoalexins. Boca Raton: CRC, 1991. p.103-143.

DILKIN, P. et al. Intoxicação experimental de suínos por fumonisinas. Ciência Rural, v.34, p.175-181, 2004.

ESPADA, Y. et al. Pathological lesions following an experimental intoxication with aflatoxin $\mathrm{B}_{1}$ in broiler chickens. Research in Veterinary Science, v.53, p.275-279, 1992. 
JAVED, T. et al. Serohematologic alterations in broiler chicks on feed amended with Fusarium proliferatum culture material or fumonisin $B_{1}$ and moniliformin. Journal of Veterinary Diagnostic Investigation, v.7, p.520-526, 1995.

KUBENA, L.F. et al. Effects of feeding Fusarium moniliforme culture material and aflatoxin singly and in combination to turkey polts. Poultry Science, v.74, p.1295-1303, 1995.

KUBENA, L.F. et al. Individual and combined effects of fuminisin $\mathrm{B}_{1}$, present in Fusarium moniliforme culture material and diacetoxyscirpenol or ochratoxin A in turkey polts. Poultry Science, v.76, p.256-264, 1997.

LEESON, S. et al. Poultry metabolic disorders and mycotoxins. Guelph: University Books, 1995. 352p.

MEIRELES, M.C.A. et al. Mycoflora of the toxic feeds associated with leukoencephalomalacia (ELEM) outbreaks in Brazil. Mycopathologia, v.127, p.183-188, 1994

MOSS, M.O. Recent studies of mycotoxins. J Applied Microbiol Symposium, v.84, p.62S-76S, 1998.

NATIONAL RESEARCH COUNCIL. Nutrient requirements of poultry. 9.ed. Washington: National Academy of Sciences, 1994. $155 \mathrm{p}$.

NORIEGA, M.L.V.C. Apuntes de hematología aviar. México Universidad Nacional Autónoma de México, 2000. 70p.

NORRED, W.P.; VOSS, K.A. Toxicity and role of fumonisins in animal diseases and human esophageal cancer. Journal of Food Protection, v.57, p.522-527, 1994

OGIDO, R. et al. Effects of prolonged administration of aflatoxin $B_{1}$ and fumonisin $B_{1}$ in laying Japanese quail.
Poultry Science, v.83, p.1953-1958, 2004

SANTIN, E. Micotoxicoses. In: BERCHIERI JÚNIOR, A.; MACARI, M. Doenças das aves. Campinas: FACTA, 2000. p. 379-388.

SAS Institute. SAS ${ }^{\circledR}$ User's Guide: Statistics. Cary, NC, 1992. 1686p

SHEPHARD, G.S. et al. Quantitative determination of fumonisins $B_{1}$ and $B_{2}$ by high-performance liquid chromatography with fluorescence detection. Journal of Liquid Chromatography, v.13, p.2077-2087, 1990.

SHOTWELL, O.L. et al. Production of aflatoxin on rice. Applied Microbiology, v.15, p.425-428, 1966.

SOARES, L.M.V.; RODRIGUEZ-AMAYA, D.B. Survey of aflatoxins, ochratoxins, zearalenone and sterigmatocystin in some Brazilian foods by using multi-toxin thinlayer chromatographic method. Journal of the Association of Official Analytical Chemists, v.72, p.22-26, 1989.

TUNG, H.T. et al. The anemia caused by aflatoxin. Poultry Science, v.54, p.1962-1969, 1975.

WEIBKING, T.S. et al. Effects of feeding Fusarium moniliforme culture material, containing known levels of fumonisin $\mathrm{B}_{1}$, on the young broiler chick. Poultry Science, v.72, p.456-466, 1993.

WEIBKING, T.S. et al. Individual and combined effects of feeding Fusarium moniliforme culture material, containing known levels of fumonisin $\mathrm{B}_{1}$, and aflatoxin $\mathrm{B}_{1}$ in the young turkey poult. Poultry Science, v.73, p.1517-1525, 1994. 